\title{
Role of Religious Orientation and Perceived Social Support in Prediction of Spiritual Well-Being of Veterans
}

\section{ART ICLE INF O}

\section{Article Type}

Descriptive Study

\section{Authors}

Sadri Damirchi E.*PhD,

Mohammadi N. ${ }^{1} M S C$

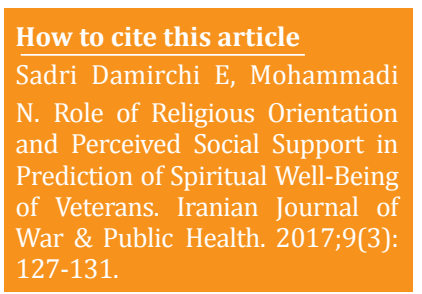

*Educational Sciences Department, Educational Sciences \& Psychology Faculty, Mohaghegh Ardabili University, Ardabil, Iran

${ }^{1}$ Educational Sciences Department, Educational Sciences \& Psychology Faculty, Mohaghegh Ardabili University, Ardabil, Iran

\section{Correspondence}

Address: Educational Sciences \& Psychology Faculty, Mohaghegh Ardabili University, Danshgah Street, Ardabil, Iran

Phone: +98 (45) 31505644

Fax: +98 (45) 33520457

araz_sadri@yahoo.com

\section{Article History}

Received: January 11, 2017

Accepted: March 14, 2017

ePublished: July 27, 2017

\section{A B S T R A C T}

Aims Veterans face many physical and psychological problems due to exposure to stressful events, which can seriously affect their spiritual well-being. The purpose of this study was to investigate the role of religious orientation and perceived social support in predicting spiritual well-being of devotees.

Instrument \& Methods In this descriptive-correlational study, 345 veterans of Rasht City, Iran, were selected by random sampling in 2016. The data collection tool was spiritual wellbeing, religious orientation, and perceived social support questionnaires. Data analysis was performed by SPSS 18 software using Pearson correlation coefficient and stepwise regression analysis.

Findings Spiritual well-being had significant positive correlations with internal religious orientation $(\mathrm{r}=0.60)$ and perceived social support $(\mathrm{r}=0.78)$ and a significant negative correlation with external religious orientation $(\mathrm{r}=-0.65 ; \mathrm{p}<0.01)$. The two variables of religious orientation and perceived social support in total explained $46 \%$ of the variance of spiritual well-being, of which $14 \%$ related to internal religious orientation, $7 \%$ to external religious orientation, and $25 \%$ to perceived social support $(\mathrm{p}<0.05)$.

Conclusion Religious orientation and perceived social support can predict the spiritual wellbeing of devotees, thus increasing the spiritual well-being of devotees by increasing internal religious orientation and perceived social support.

Keywords Spiritual Well-Being; Religious Orientation; Social Support; Veterans

\section{I T A T I O N L I N KS}

[1] The effectiveness of mindfulness training on psychological symptoms in veterans with posttraumatic stress ... [2] Conceptual definition and operationalization of spiritual health: A ... [3] Effects of bereavement life review on spiritual well-being and ... [4] Spiritual well-being in cancer patients under ... [5] Assessing religious care needs of patients in ... [6] Identity Styles and Religious ... [7] Relationship between religious activities and spiritual health with glycemic control in patients ... [8] The relationship between religious orientation and psychological wellbeing in ... [9] The role of coping styles and social support for diagnosing students with high and low test... [10] The role of perception and perceived social support in the ... [11] Social support, world assumptions, and exposure as predictors of anxiety and quality of life following ... [12] Structural relationship between hope with attachment style, life regard, social support, selfefficacy and mastery goal orientation ... [13] Does social support help ... [14] The roles of social support in helping chinese women with antenatal depressive and anxiety symptoms cope with ... [15] The effect of spirituality and social support on mental health ... [16] Psychometric characteristics of spiritual well-being among students in ... [17] Relationship between religious orientation and psychological adjustment ... [18] The multidimensional scale of perceived social support: A confirmation ... [19] Relationship between perceived social support and risk of postpartum depression ... [20] Religion, mental health and loneliness: Examine the relationship between the level of religiosity and mental health with loneliness ... [21] Relationship between religious orientation and psychological well-being ... [22] Psychosocial mediation of religious coping styles: A study of short-term psychological distress following cardiac ... [23] Relational spirituality and depression in adolescent ... [24] The role of religion on mental health of university students in Ilam ... [25] You can't always get what you want, but can you get what you need? Personality traits and social support in ... [26] Social support, stress, health, and academic success in Ghanaian adolescents: A path ... [27] A study on the relationship between different dimensions of perceived social support and different aspects of ... [28] NFamilies with children who have autism spectrum disorders: Stress and ... 
امروزه توجه به بُعد سلامت معنوى جانبازان مورد توجه جدى قرار مُاري

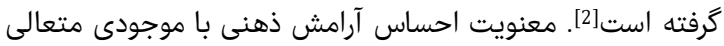

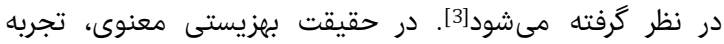

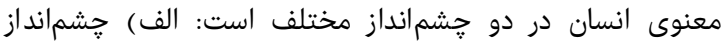

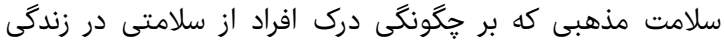

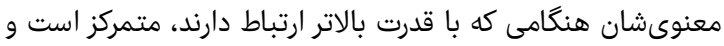

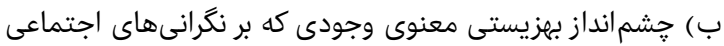

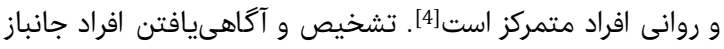

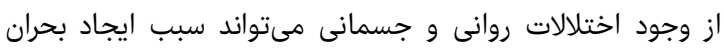

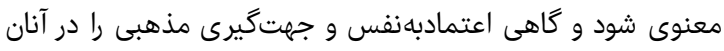

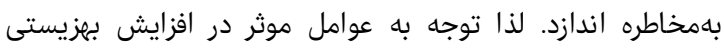
معنوى جانبازان ضرورت داردا.

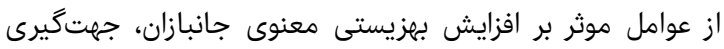

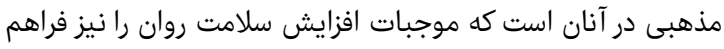

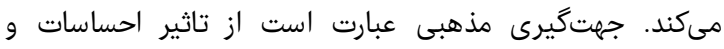

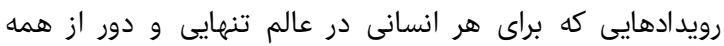

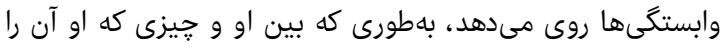

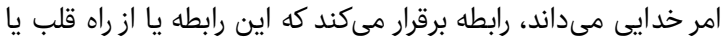

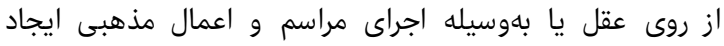

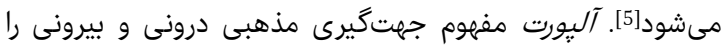

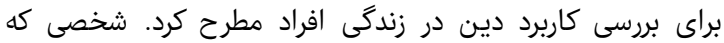

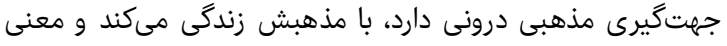

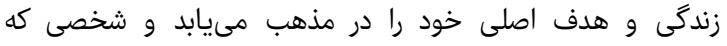

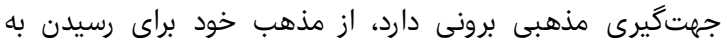

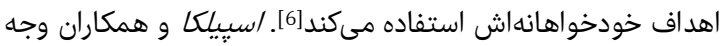

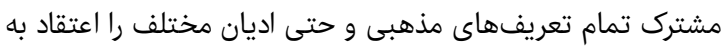

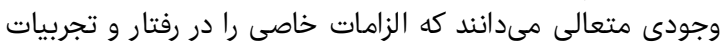

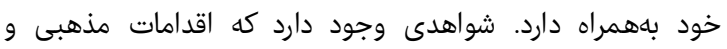

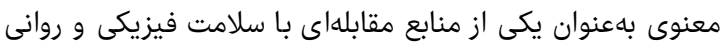

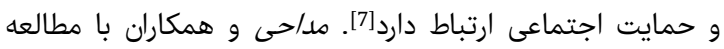

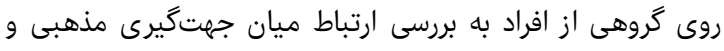

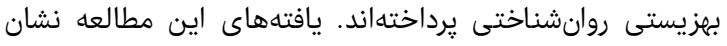

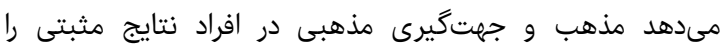

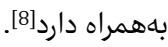

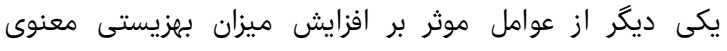

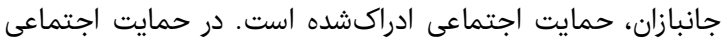

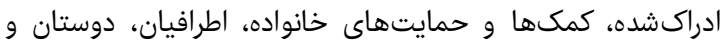

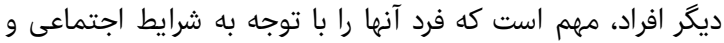

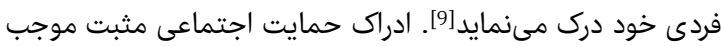

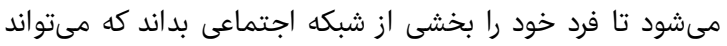

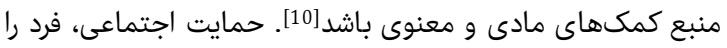

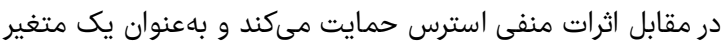

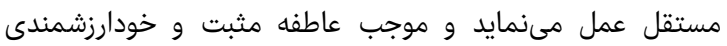

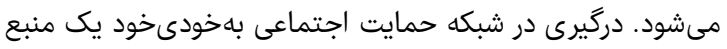

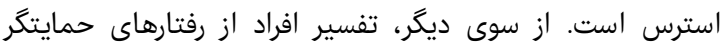

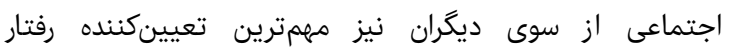

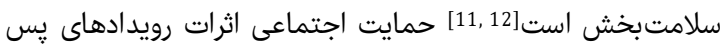

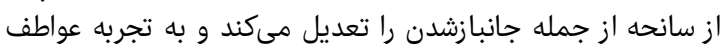

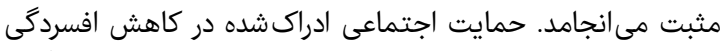

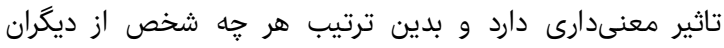

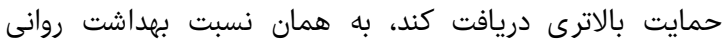

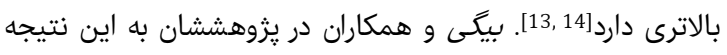

دوره 9، شماره س، تابستان عوسا

\section{نقش جهت راعيرى مذهبى و و حمايت اجتماعى ادراكشده در ييشبينى بهزيستى معنوى جانبازان}

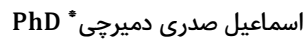

كروه علوم تربيتى، دانشكده علوم تربيتى و روان شناسى، دانشكاه محقق اردبيلى،

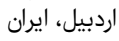

MSc نسيم محمدى ايرنى

كَروه علوم تربيتى، دانشكده علوم تربيتى و روان شناسى، دانشكاه محقق اردبيلى، ارئى

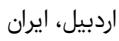

קكيده اهداف: جانبازان بهدليل مواجهه با حوادث استرسزاي جنگَ، با مشكلات

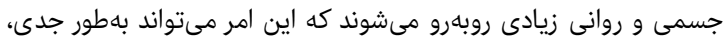

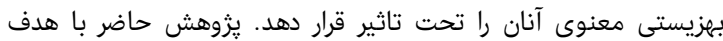

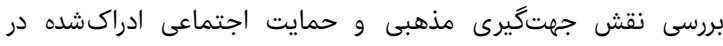

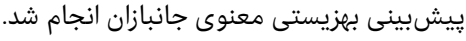

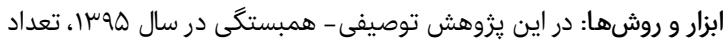

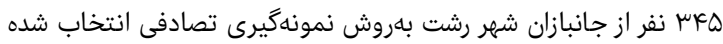

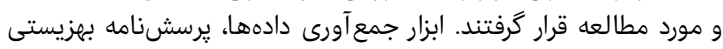

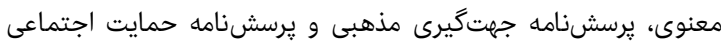

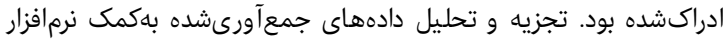
SPSS 18

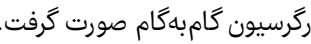

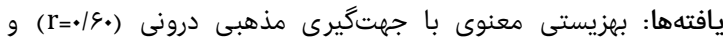

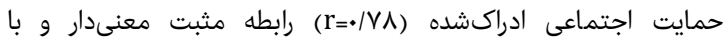

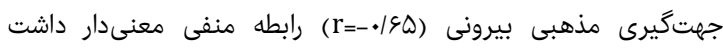

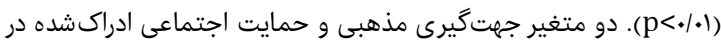

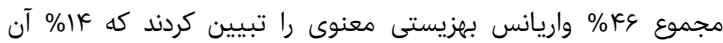

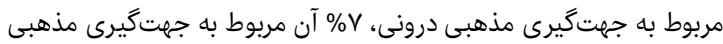

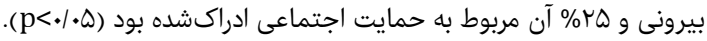

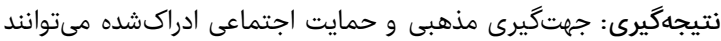

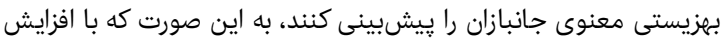

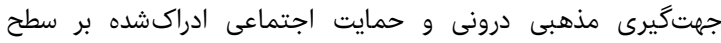

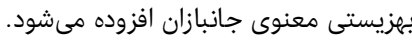
كليدوازمها: بهزيستى معنوى جنبازي جهتئيرى مذهبى، حمايت اجتماعى، جانبازان

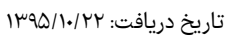

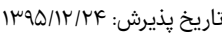

araz_sadri@yahoo.com :نويسنده مسئول:

آسيبديدگى ناشى از جنگ، از ديرباز براى تمامى ملل از جمله

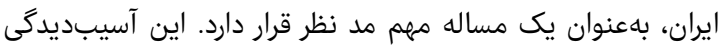

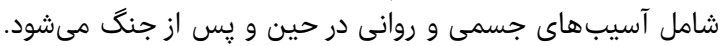

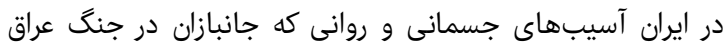

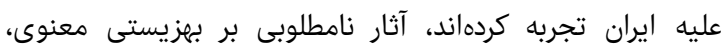

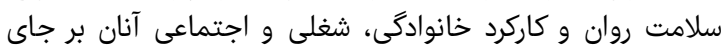

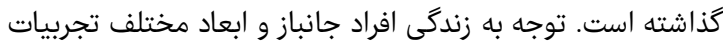

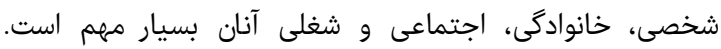

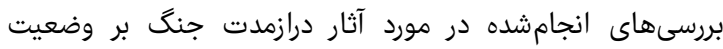

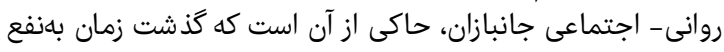

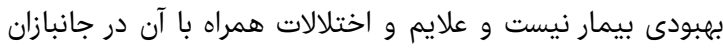

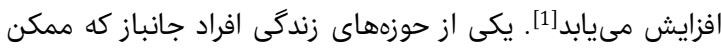

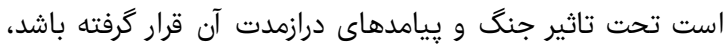
بهزيستى معنوى جانبازان است. فصل نامه علمى - يخروهشى طب جانباز 


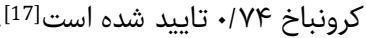

س- مقياس حمايت اجتماعى ادراكشده جندبعدى (MSPSS)

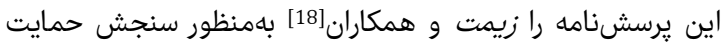

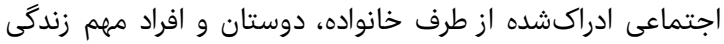

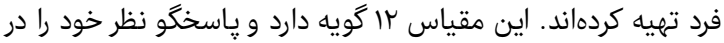

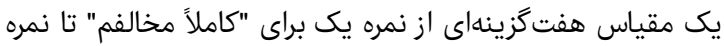

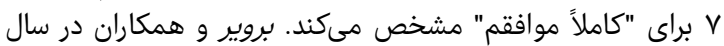

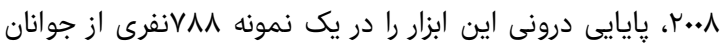

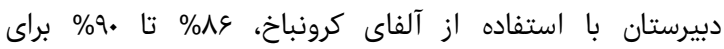

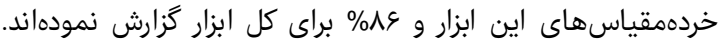

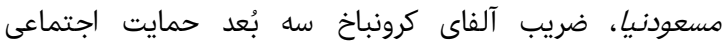

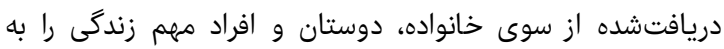

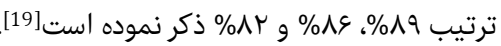

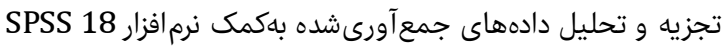

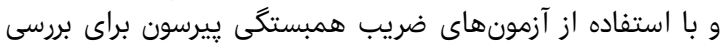

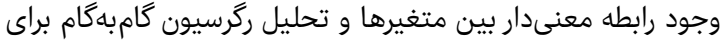

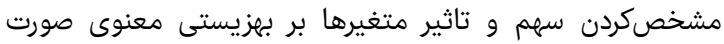

\section{يافتهها}

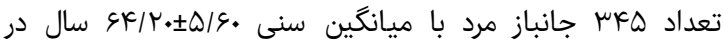

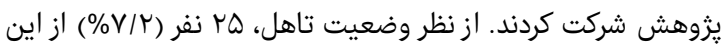

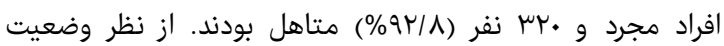

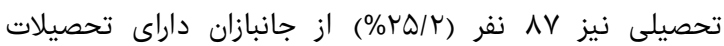

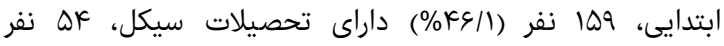

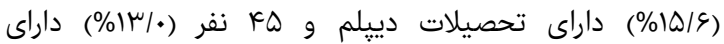
تحصيلات بالاتر ازدييلم بودند. تجاني

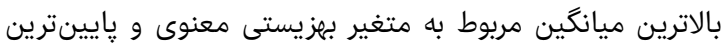

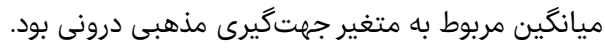

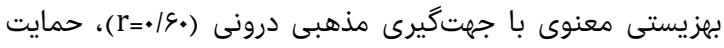

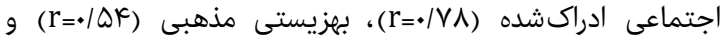

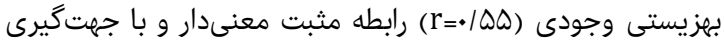

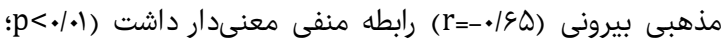

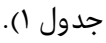

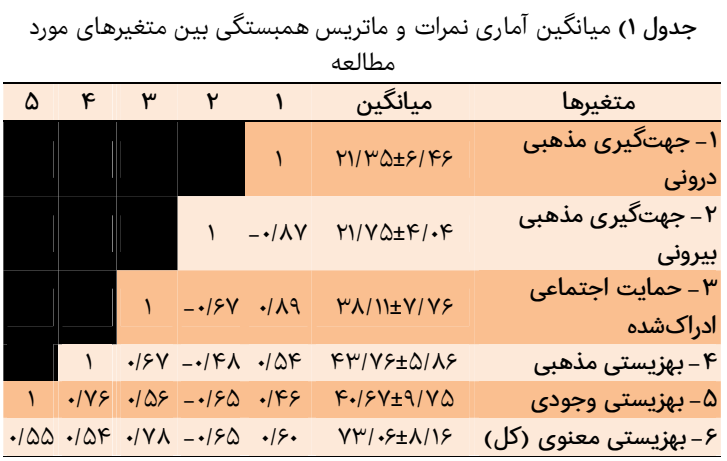

دو متغير جهتگيرى مذهبى و حمايت اجتماعى ادراكشده در كر

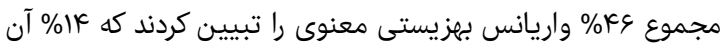

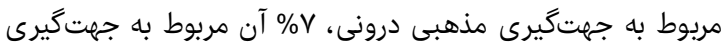

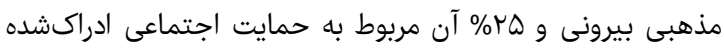

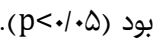

رسيدند كه حمايت اجتماعى و فعاليتهاى اجتماعى مذهبى

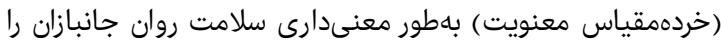

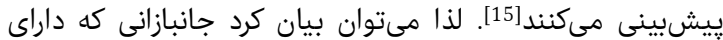

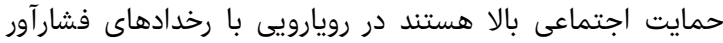

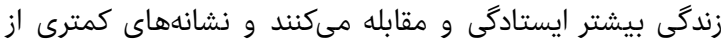

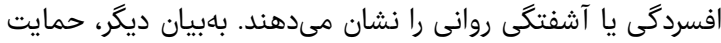

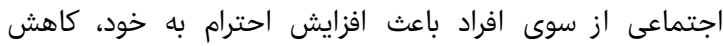

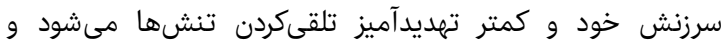

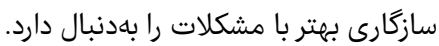

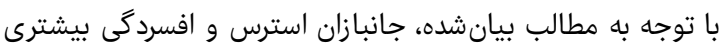

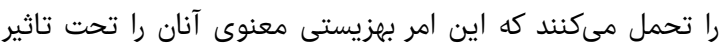

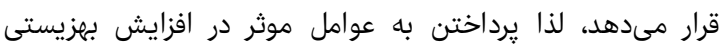

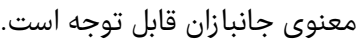

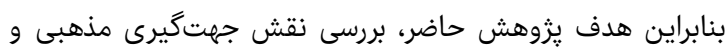

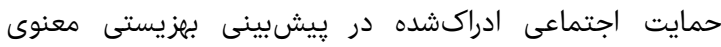

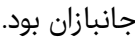

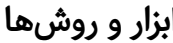

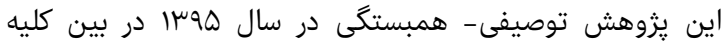

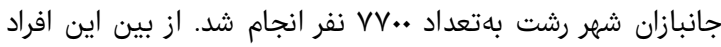

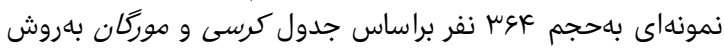

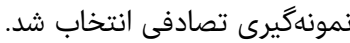

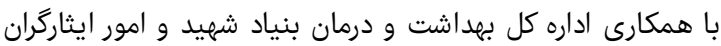

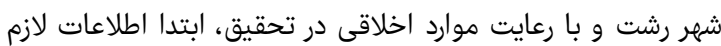

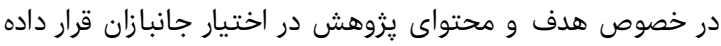

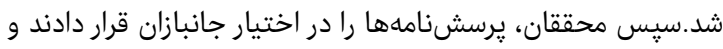

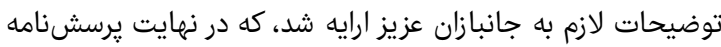

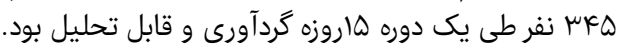

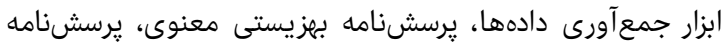

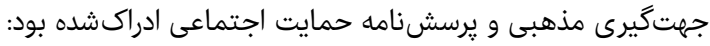

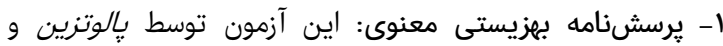

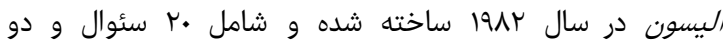

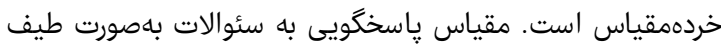

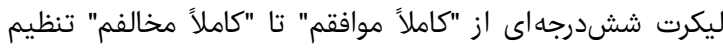

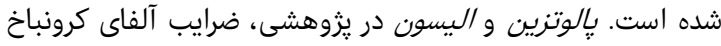

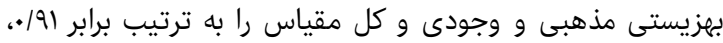

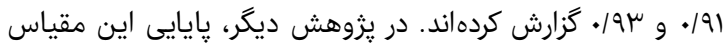

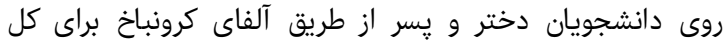

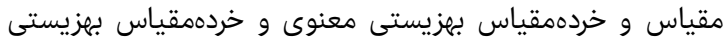

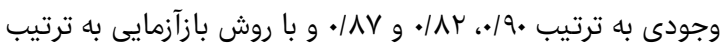

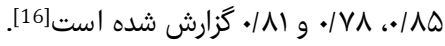
r- ״رسشنامه جهت

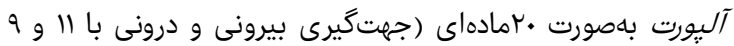

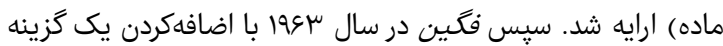

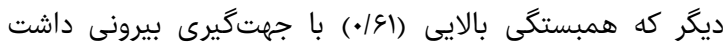

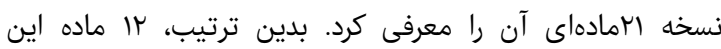

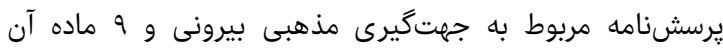

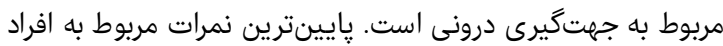

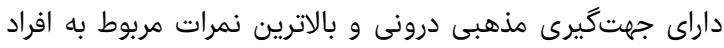

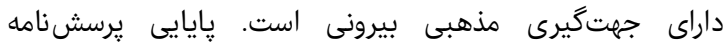

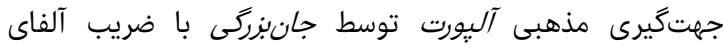




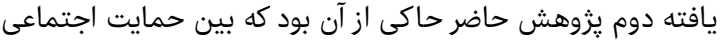

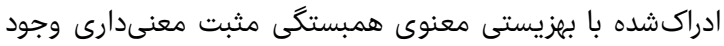

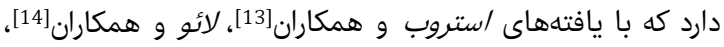

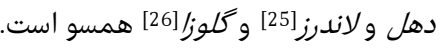

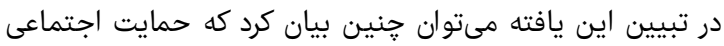

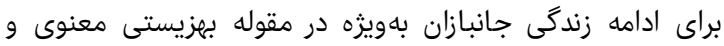

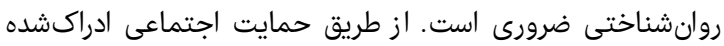

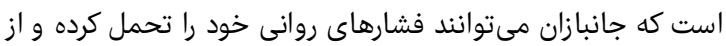

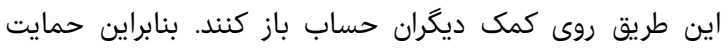

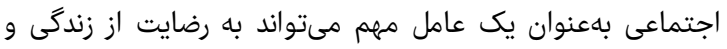

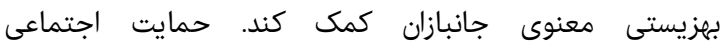

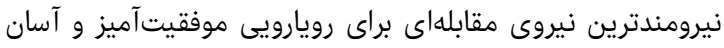

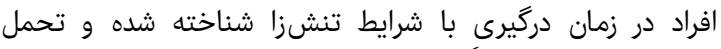

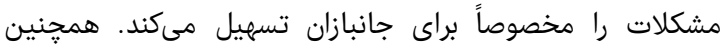

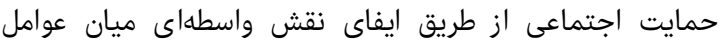

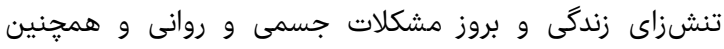

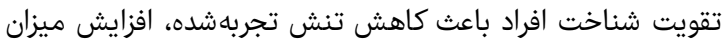

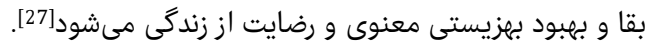

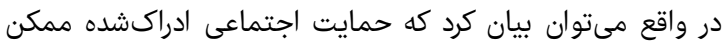

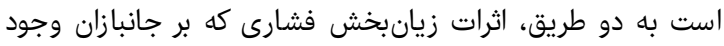

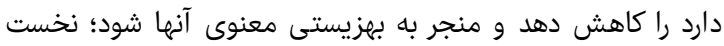

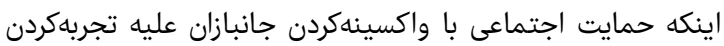

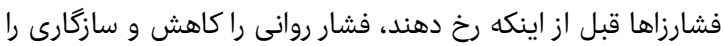

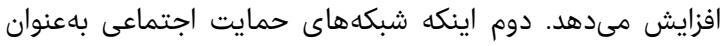

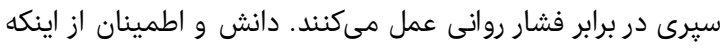

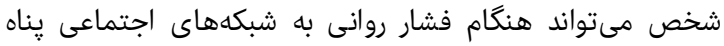

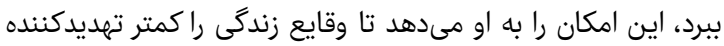

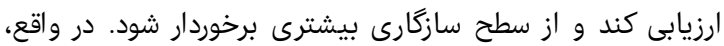

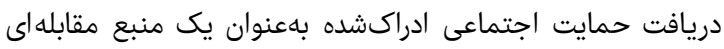

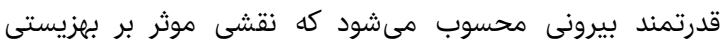

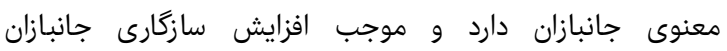
مى شود [28]. بهور خلاصه، بهنظر مىرسد جانبازانى كه يس از تحمل آسيبهاى

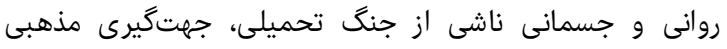

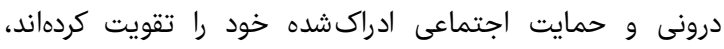

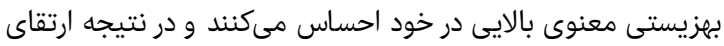

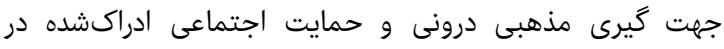

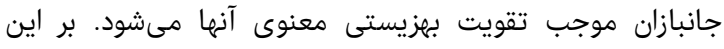

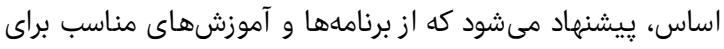

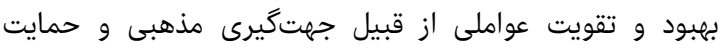

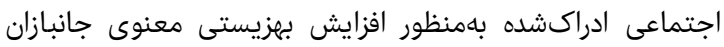

استفاده شود.

از جمله محدوديتهاي اين يزوهش، انتخاد انتخاب نمونه صرفاً از ميان

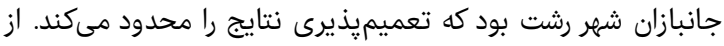

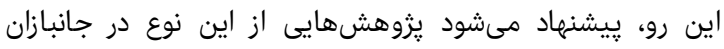
شهرهاى ديگر انجام شود.

\footnotetext{
نتيجه

جهتگيرى مذهبى و حمايت اجتماعى ادراكشده مى مئوانند

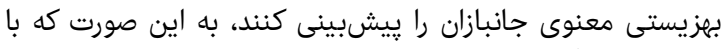

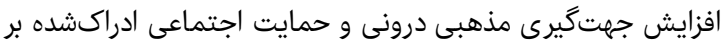

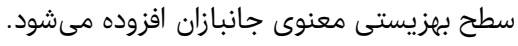

دوره 9، شماره س، تابستان عوس1
• سا اسماعيل صدرى دميرجى و نسيم محمدى ـ.

با توجه به اهميت بهزيستى معنوى بهخصوص براى جانبازان،

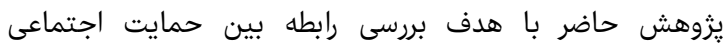
ادراكشده و جهت

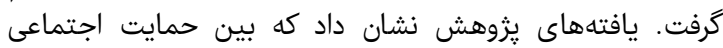

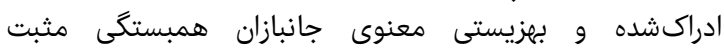

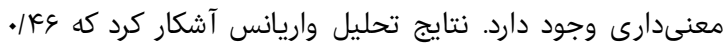

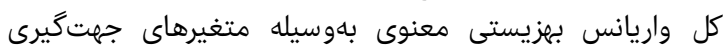

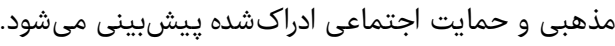

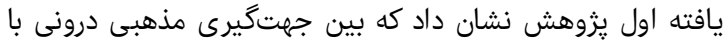

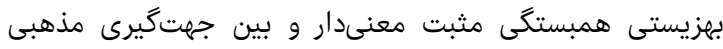

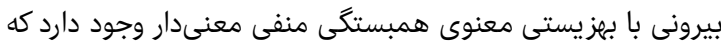

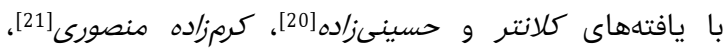

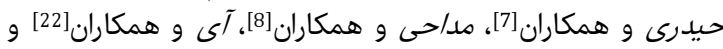
دسروسيروس و ميلر [23] همسو است مدارئ

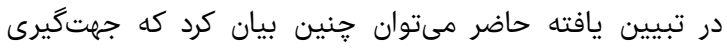

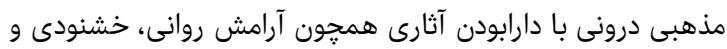

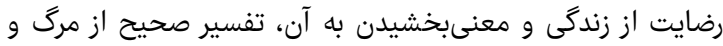

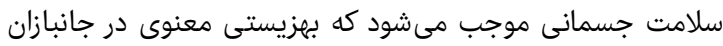

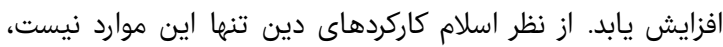

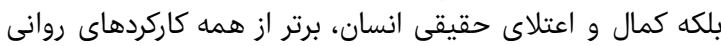

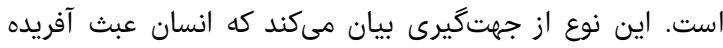

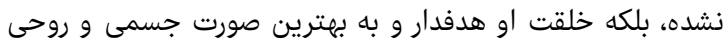

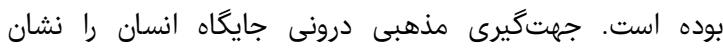

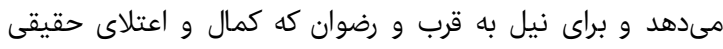

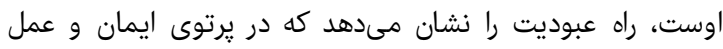

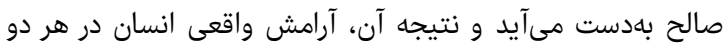

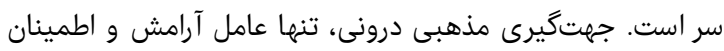

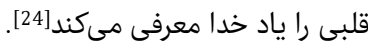

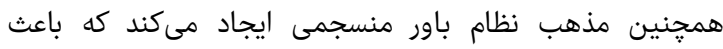

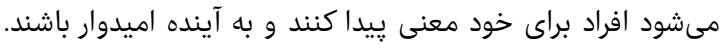

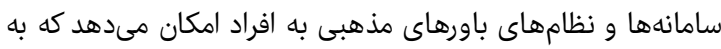

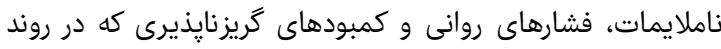

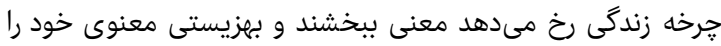

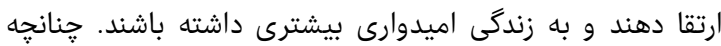

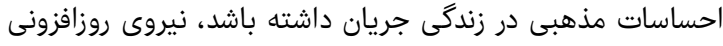

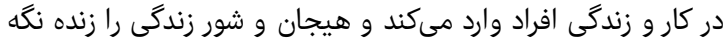

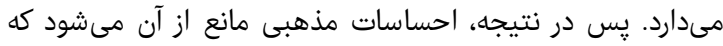

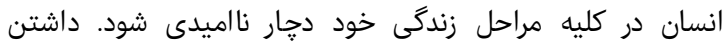

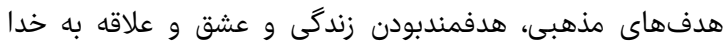

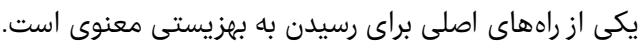

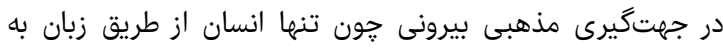

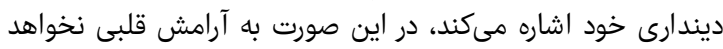

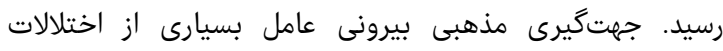

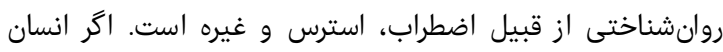

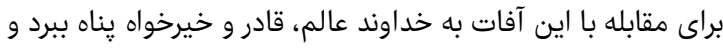

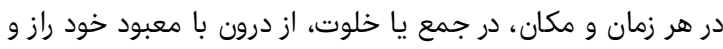

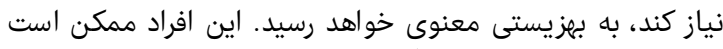

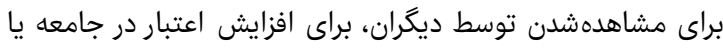

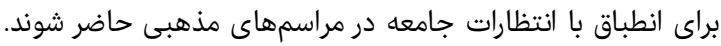

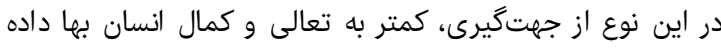

مىشود[8].

فصل نامه علمى - يخروهشى طب جانباز 
نقش جهت

Psychol Health. 2013;7(1):1-10. [Persian]

13- Strobe W, Zech E, Strobe MS, Abakoumkin G. Does social support help in bereavement?. J Soc Clin Psychol. 2005;24(7):1030-50.

14- Lau Y, Wong DF, Wang Y, Kwong DH, Wang Y. The roles of social support in helping chinese women with antenatal depressive and anxiety symptoms cope with perceived stress. Arch Psychiatr Nurs. 2014;28(5):305-13.

15- Beigi A, Ahmadi Tahor Soltani M, Mohammadifar M, Najafi M. The effect of spirituality and social support on mental health of veterans. J Mil Psychol. 2012;3(11):119. [Persian]

16- Dehshiri Gh, Sohrabi F, Jafari I, Najafi M. Psychometric characteristics of spiritual well-being among students in psychological studies. Psychol Studies. 2008;4(3):129-44. [Persian]

17- Khodapanahi M, Khaksarbaldaji M. Relationship between religious orientation and psychological adjustment in students. J Psychol Relig. 2005;9(3):31020. [Persian]

18- Dahlem NW, Zimet GD, Walker RR. The multidimensional scale of perceived social support: A confirmation study. J Clin Psychol. 1991;47(6):756-61.

19- Masoudnia E. Relationship between perceived social support and risk of postpartum depression disorder. Iran J Nurs. 2011;24(70):8-18. [Persian]

20- Kalantar A, Hosseini Zadeh S. Religion, mental health and loneliness: Examine the relationship between the level of religiosity and mental health with loneliness (study case: Tehran citizens). J Appl Soc. 2015;26(4):2544. [Persian]

21- Azmodeh P, Shahidi P, Danesh E. Relationship between religious orientation and psychological wellbeing and happiness. Psychology. 2007;11(1):60-74. [Persian]

22- Ai AL, Park CL, Huang B. Rodgers W, Tice TN. Psychosocial mediation of religious coping styles: A study of short-term psychological distress following cardiac surgery. Personal Soc Psychol Bull. 2007;33(6):867-82.

23- Desrosiers A, Miller L. Relational spirituality and depression in adolescent girls. J Clin Psychol. 2007;10(63):1021-37.

24- Kheydani L. The role of religion on mental health of university students in Ilam Province. Innov Manag Educ. 2011;6(3):63-82. [Persian]

25- Dehle C, Landers JE. You can't always get what you want, but can you get what you need? Personality traits and social support in marriage. J Soc Clin Psychol. 2005;24(7):1051-76.

26- Glozah FN, Pevalin DJ. Social support, stress, health, and academic success in Ghanaian adolescents: A path analysis. J Adolesc. 2014;37(4):451-60.

27- Ghaedi Gh, Yaaghoobi H. A study on the relationship between different dimensions of perceived social support and different aspects of wellbeing. Armaghan-eDanesh. 2008;13(2):69-81. [Persian]

28- Meadan H, Halle JW, Ebata AT. Families with children who have autism spectrum disorders: Stress and support. Except Child. 2010;77(1):27-38.

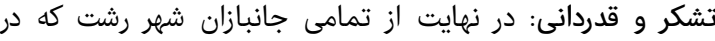

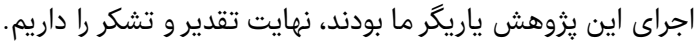

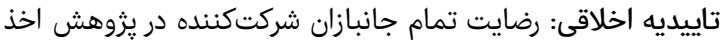

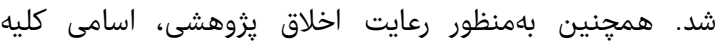

$$
\begin{aligned}
& \text { جانبازان بهصورت كد وارد شد. } \\
& \text { تعارض منافع: موردى از سوى نويسندكان اعلام إندان نشده است. }
\end{aligned}
$$

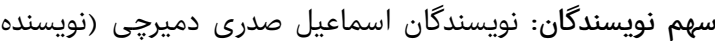

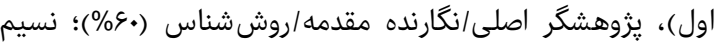

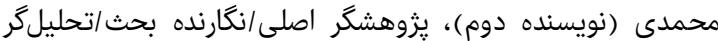

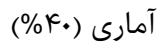

$$
\begin{aligned}
& \text { منابع مالى: موردى از سوى نويسندگًان اعلام نشده است. }
\end{aligned}
$$

منابع

1- Azad Marzabadi E, Moqtadaee K. The effectiveness of mindfulness training on psychological symptoms in veterans with posttraumatic stress disorder. Int J Behav Sci. 2014;7(1):67-74.

2- Abbasi M, Azizi F, Shamsi Gooshki E, Naseri Rad M, Akbari Lakeh M. Conceptual definition and operationalization of spiritual health: A methodological study. Med Ethics J. 2013;6(20):11-44. [Persian]

3- Ando M, Morita T, Miyashita M, Sanjo M, Kira H, Shima $\mathrm{Y}$ Effects of bereavement life review on spiritual wellbeing and depression. J Pain Symptom Manage. 2010;40(3):453-9.

4- Habibi A, Savadpour MT. Spiritual well-being in cancer patients under chemotherapy. J Health Care. 2011;13(3):16-20. [Persian]

5- Zand S, Rafiei M. Assessing religious care needs of patients in hospital. Teb va Tazkiyeh. 2013;19(4):19-21. [Persian]

6- Sargazi M, Nikmanesh Z. Identity Styles and Religious Self-Regulatio. Ravanshenasi va Din. 2013;6(2):19-21. [Persian]

7- Heidari S, Raisi M, Ahmari-Tehran H, Khorami-Rad A. Relationship between religious activities and spiritual health with glycemic control in patients with diabetes. Iran J Nurs. 2013;26(81):78-87. [Persian]

8- Maddahi ME, Samadzade M, Kekhay Farzaneh MM. The relationship between religious orientation and psychological well-being in students. Psychol Educ J. 2011;2(1):53-63. [Persian]

9- Faraji A, Ariapouran S, Abdi A. The role of coping styles and social support for diagnosing students with high and low test anxiety. Educ Psychol. 2010;6(4):4768. [Persian]

10-Salimi A, Jokar B, Nikpor R. The role of perception and perceived social support in the internet use. Res Soc Psychol J. 2009;5(3):81-102. [Persian]

11- Grills-Taquechel AE, Littleton HL, Axsom D. Social support, world assumptions, and exposure as predictors of anxiety and quality of life following a mass trauma. J Anxiety Disord. 2011;25(4):498-506.

12- Ahmadi Tahour Soltani M, Karaminia R, Ahadi $\mathrm{H}$, Moradi AR. Structural relationship between hope with attachment style, life regard, social support, selfefficacy and mastery goal orientation in students. J Res 